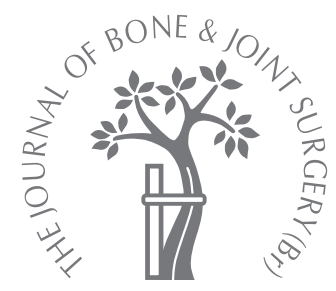

\title{
Two-stage revision arthroplasty of the hip for infection using an interim articulated Prostalac hip spacer
}

\author{
A 10- TO 15-YEAR FOLLOW-UP STUDY
}

\begin{abstract}
G. S. Biring,
T. Kostamo,

D. S. Garbuz,

B. A. Masri,

C. P. Duncan
\end{abstract}

From the University

of British Columbia,

Vancouver, Canada

We report the outcome at ten to $\mathbf{1 5}$ years of two-stage revision for hip infection in 99 patients using the Prostalac articulated hip spacer system.

All the patients were contacted to determine their current functional and infection status using the Oxford-12, Short form-12, and Western Ontario and McMaster University Osteoarthritis Index questionnaires. A total of 11 of the 99 patients had a further infection, of whom seven responded to repeat surgery with no further sequelae. The mean interval between the stages was five months ( 1 to 36 ). We were able to review 48 living patients, with a mean age of 72 years (46 to 86$), 34(71 \%)$ of whom provided health-related quality-oflife outcome scores.

The mean follow-up was 12 years (10 to 15). The long-term success rate was $89 \%$ and with additional surgery this rose to $96 \%$. The mean global Western Ontario and McMaster University Osteoarthritis Index score was 80.6 (SD 18.3). The mean Oxford-12 score was 74.0 (SD 22.3), and the mean Short form-12 score was 53.1 (SD 9.4) (mental) and 33.5 (SD 13.5) (physical). The mean satisfaction score was 90.5 (SD 15.3).

Two-stage revision for hip infection using a Prostalac interim spacer offers a predictable and lasting solution for patients with this difficult problem.

Deep-seated infection after total hip replacement (THR) has grave consequences and is difficult to treat. The prevalence of infection after THR increases with time. ${ }^{1}$ The success of one- or two-stage management decreases after a number of years because of reinfection $^{2,3}$ and the emergence of resistant organisms. ${ }^{4}$ There are a number of options for the treatment of chronic infection of a THR. While there have been reports of a successful outcome after immediate reimplantation, ${ }^{5,6}$ the best results have been obtained when this was delayed by at least six weeks with rates of control of over $90 \% .^{7-9}$ Our study focuses on the two-stage approach to treatment with the use of an articulated spacer between the stages. This spacer was made of antibiotic-loaded acrylic cement and was named Prostalac (prosthesis of antibiotic-loaded acrylic cement (Depuy, Warsaw, Indiana)).

Our primary aim was to determine whether the use of this system along with the other principles of management was associated with the long-term eradication of infection. We also assessed the quality of life and satisfaction in this group of patients and determined the survivorship of the revision THR.

\section{Patients and Methods}

We identified from our database 103 patients who had been treated using a Prostalac hip spacer at our centre between November 1989 and June 1996. The approval of the Institutional Review Board was obtained to enrol these patients in the study. Four had not undergone a second-stage revision THR and therefore were excluded. Two of these died between the stages because of advanced age with unrelated causes and no evidence of persisting infection. One other patient was so pleased with the interim prosthesis that he declined further surgery. The fourth patient was deemed to be medically unfit for any further surgery. This left 99 patients (52 men and 47 women) in the series with a mean age at the time of follow-up of 64 years (28 to 86). A total of 92 patients were followed up. Seven were lost to follow-up, 44 had died and 48 were still alive for analysis. The mean follow-up was 12 years (10 to 15 ).

Of the seven patients lost to follow-up, two had a further infection. These were included in our results. One failed the two-stage exchange, underwent resection arthroplasty and eventual re-implantation and was infection-free at last follow-up. One required a repeat first-stage procedure and then became infection-free. 
All the patients were contacted at the time of review for a complete independent assessment as to whether they had a recurrence of the infection, had undergone further revision surgery, were awaiting further surgery or had any tests for suspected infection. Failure of the treatment was defined as recurrence of infection with a proven positive culture or any positive response to the above questions. The clinic and hospital notes of the deceased patients were carefully reviewed, the next of kin interviewed and, if there was any doubt, the family practitioner was contacted to ensure that no further infection or surgery had occurred.

The following quality-of-life measures were obtained for each patient: the Western Ontario McMaster (WOMAC) osteoarthritis index ${ }^{10}$ the Oxford-12, ${ }^{11}$ the Short-form (SF)- $12^{12}$ and the University of California Los Angeles (UCLA) activity scale. ${ }^{13}$ A satisfaction questionnaire was used which was also applicable to the second-stage revision patients. ${ }^{14}$ The Charnley class was used for the assessment of comorbidity as follows: class A, ipsilateral joint replacement; class B1, ipsilateral replacement with degenerative change in the contralateral hip; class B2, both hips replaced; and class $\mathrm{C}$, multiple joint disease or other disabilities leading to difficulties in walking. ${ }^{15}$

Operative technique. The Prostalac system is relatively simple and allows the intra-operative manufacture and implantation of a temporary replacement using bone cement to which powdered antibiotics are added, of a type and dose considered to be optimal for the patient under treatment. The acetabular component is a triflanged polyethylene cup with a snap-fit mouth to constrain the femoral head. It is orientated in $45^{\circ}$ of lateral and $20^{\circ}$ of anterior opening within a bed of doughy cement which has been hand-pressurised within the native acetabulum after it has been thoroughly cleansed and shallow key-holes created. The aim is to achieve secure fixation without deep interdigitation of cement into bone. The femoral side is a system of standard and long stems within a sleeve of antibiotic-loaded acrylic cement. This sleeve is constructed with a system of moulds of variable widths and lengths. The size and length are chosen based on the needs of the patient under treatment. This preset composite of antibiotic cement-coated stem is introduced as a press-fit into the cleansed and prepared femur achieving stable vertical and torsional stability in most cases. If necessary, a cuff or collar of antibiotic cement is added to achieve stability, particularly in patients with a markedly deficient proximal femur. In addition to the stability of this temporary fixation, the stem and the acetabular component are easily extractable thus making the second-stage operation less difficult (Fig. 1).

Parenteral antibiotics were administered based on the culture and sensitivity profile of the infecting organism. The antibiotic used in the cement typically included $1 \mathrm{~g}$ to $1.5 \mathrm{~g}$ of vancomycin (Vacocin; Eli Lilly, Canada Inc, Scarborough, Ontario) and $2.4 \mathrm{~g}$ to $3.6 \mathrm{~g}$ of tobramycin (Nebcin; Eli Lilly). Occasionally, penicillin-G (Novopharm Ltd., Toronto, Ontario) or another antibiotic based on the

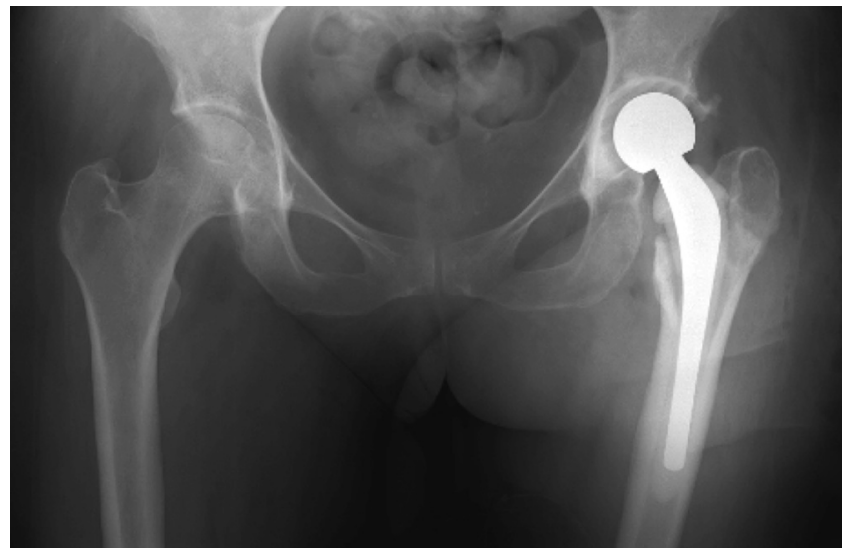

Fig. 1

Radiograph showing the first-stage Prostalac short-stem spacer in situ.

sensitivity profiles of organisms cultured was added. The wound was closed in layers without the drains to avoid the removal of antibiotics from the periprosthetic space.

The patients were allowed to mobilise partially weightbearing on crutches. In most intravenous antibiotics were continued for six weeks. Typically, the laboratory assessments of the inflammatory markers (ESR and white cell count) were repeated at the time at which the antibiotics were stopped and four weeks later. A hip aspiration for aerobic and anaerobic culture and sensitivity of the aspirated fluid was performed typically at ten weeks after the first stage (four weeks after the discontinuation of the antibiotics).

At the second-stage the Prostalac system was removed, usually with ease, and fixation of the implant completed along with supplementary reconstruction as required. Intravenous antibiotics were continued until the final intra-operative culture results were available, usually at five days. The rehabilitation was tailored to the needs of the reconstruction and the patient as well as being based on the intra-operative findings and post-operative radiographs.

\section{Results}

The indication for a two-stage procedure included prosthetic infections in 92 patients, sepsis in a native hip in five and an infected internal fixation device used for proximal femoral fracture in two. The causative organisms are listed in Table I. The common staphylococci and streptococci were seen with a substantial proportion of patients $(20 \%)$ having mixed organisms on culture. Mycobacterium was cultured from one and in five no organisms were isolated, but at that time of operation pus was encountered and the mean ESR was 92.0 (SD 33.7) with no other cause identified. All had evidence of acute inflammation (greater than five polymorphonuclear leucocytes per high power field) on histological analysis, consistent with infection. There was a methicillin-resistant species in ten patients. One was in the 
Table I. Microbiological flora causing infection

\begin{tabular}{ll}
\hline Organism & Number \\
\hline Staph. epidermidis (9 methicillin-resistant) & 22 \\
Staph. aureus (1 methicillin-resistant) & 22 \\
Multiple organisms (staphylococcus and streptococcus) & 20 \\
Streptococcal species & 12 \\
Other gram-negative organisms & 10 \\
Other staphylococcus species & 4 \\
Escherischia coli & 3 \\
Mycobacterium tuberculosis & 1 \\
No organism identified & 5 \\
\hline
\end{tabular}

group lost to follow-up while the remainder were successfully treated by the two-stage procedure.

Palacos R bone cement (Zimmer, Warsaw, Indiana) was used in every case and a single or combination of antibiotics added. This included vancomycin and tobramycin in 86 patients $(87 \%)$, tobramycin and penicillin $G$ in six, tobramycin alone in three, vancomycin and penicillin $G$ in two, streptomycin (Pfizer Canada, Point Claire, Quebec) for the treatment of infection with Mycobacterium tuberculosis in one, and ceftizoxime (SmithKline Beecham Pharmaceuticals, Oakville, Ontario) for treatment of infection with Bacillus species in one.

Assessment of comorbidities showed that 69 patients $(70 \%)$ were in Charnley class C, $13(13 \%)$ in class B and $17(17 \%)$ were class A.

The mean time between the stages was five months (1 to 36). All hip aspirations were negative before the second stage. None of the hips appeared to be infected at the second stage and the reconstruction proceeded as planned. The same approach was used for both stages and was based on the preference of the surgeon and anatomical consideration of the patient. In 51 patients the direct lateral approach was used, in 30 a trochanteric osteotomy, in one the MacFarland-Osborne approach ${ }^{16}$ and in 17 the posterior approach. A total of 19 patients required an extended trochanteric osteotomy at the first stage to remove components. The trochanteric slide was used in three patients.

The femoral components implanted consisted of 14 segmental proximal femoral placements, four allograftprosthetic composites, 62 cemented components, and 19 uncemented components. On the acetabular side 96 were uncemented components (including five bipolar components), and three were cemented.

Clinical outcome. A total of 11 patients had re-infection giving an initial eradication rate of $89 \%$. Seven of these patients responded to revision surgery and became infection-free in the long term (mean 11.4 years (10 to 14)). Five of these had a recurrent infection within one year of the initial second-stage operation and two had later recurrent infections at 27 and 36 months. There were four failures. Two patients required resection arthroplasty, one had a hip disarticulation after eventual failure of treatment and severe bone loss and one illicit drug-dependent, immune- compromised paraplegic patient developed osteomyelitis with an intermittently draining sinus which was treated with chronic suppressive oral antibiotics and dressings. Of the group of four final failures, all had a late recurrent infection at 2.5, 4.5, 6, and 6 years, respectively. Of the seven patients lost to follow-up two had a further infection and all were included in our results. If the other five were deemed to be failures then in the worst-case scenario the infection eradication rate was $84 \%$.

In the group of 11 patients who failed the initial twostage revision, multiple organisms were isolated in seven. Four had gram-negative rods and the others a combination of Staphylococcus aureus, Staph. epidermidis and $\beta$ haemolytic/streptococcus. In one no organisms were isolated. Five had recurrence of the organisms which had been isolated at the time of the pre-operative investigation at the first stage (Table II). In this group of 11 patients, three had proximal femoral replacements, two proximal femoral composite grafts, three hybrid revision hip replacements (cemented acetabular and uncemented femoral components), two cemented prostheses and one an uncemented implant.

Further revision surgery was required for reasons not related to infection in nine patients giving a rate of reoperation of $9 \%$. Four patients had revision for aseptic loosening at a mean time of 7.6 years (5 to 11) showing a survivorship rate of $96 \%$ during the period of study if using this endpoint. Three patients had a recurrent dislocation, one of which was combined with a periprosthetic fracture with no further recurrence of dislocation. One patient underwent a revision for a periprosthetic fracture alone and one for removal of cable and claw. Of the four patients who developed aseptic loosening, three had revision of both components and one of the acetabular component only. Of these, three acetabular components were cemented and one uncemented. Of the femoral components, two were cemented and two uncemented. There was no evidence of nonunion of the trochanter, the proximal femoral allografthost bone junction or the extended trochanteric osteotomy. Quality-of-life outcome and patient satisfaction. Quality-oflife outcome data were available for 34 of the 48 patients who were still alive. With reference to the Charnley class the distribution of the patients who returned the questionnaires was similar with $26(76.5 \%)$ being in class C, $3(8.8 \%)$ in class B and $5(14.7 \%)$ in class A. Thus, the sample was representative of the group as a whole. Figures 2 and 3 show the quality-of-life outcome scores including the WOMAC, the Oxford-12, the SF-12 (physical and mental score), and the overall satisfaction. Good outcomes were achieved with results comparable with those in patients undergoing revision hip replacement for other indications in our institution. The overall satisfaction was high with a mean satisfaction score of 90.5 (SD 15.3). The mean UCLA activity score was $4.1 \pm 1.8$ ( 1 to 8 ) which indicated that patients regularly participated in mild activities such as walking, limited housework and limited shopping. 
Table II. Details of organisms isolated at the time of first-stage Prostalac revision, re-infection organisms, time to re-infection and implants used at the second-stage total hip replacement

\begin{tabular}{|c|c|c|c|c|}
\hline Case & First-stage organism & Re-infection organism & $\begin{array}{l}\text { Time to re-infection } \\
\text { (mths) }\end{array}$ & $\begin{array}{l}\text { Type of second-stage } \\
\text { prosthesis }^{*}\end{array}$ \\
\hline 1 & Staph. epidermidis and Enterococcus faecalis & Staph. epidermidis and Enterococcus faecalis & 1.0 & Hybrid \\
\hline 2 & $\begin{array}{l}\text { Klebsiella pneumoniae and group-B } \\
\text { streptococcus }\end{array}$ & E. coli & 2.5 & PFAC \\
\hline 3 & $\begin{array}{l}\text { Staph. epidermidis and } \beta \text {-haemolytic } \\
\text { streptococcus }\end{array}$ & Staph. epidermidis & 3.0 & PFR \\
\hline 4 & Staph. aureus & Staph. aureus & 4.0 & Hybrid \\
\hline 5 & No organism & No organism & 5.6 & PFR \\
\hline 6 & Staph. aureus and Corynebacterium jeikeium & group-B streptococcus & 12.0 & Uncemented \\
\hline 7 & Staph. epidermidis & Coagulase-negative Staphylococci & 18.0 & PFAC \\
\hline 8 & $\begin{array}{l}\text { Peptostreptococci, Enterococcus spp and } \\
\text { Staph. aureus }\end{array}$ & $\begin{array}{l}\text { Peptostreptococci, Enterococcus spp and Staph. } \\
\text { aureus }\end{array}$ & 30.0 & Cemented \\
\hline 9 & Staph. epidermidis & Proprionibacterium spp & 54.0 & PFR \\
\hline 10 & $\begin{array}{l}\text { Staph. aureus, coagulase-negative } \\
\text { Staphylococci, Streptococcus pneumoniae }\end{array}$ & Staph. aureus & 72.0 & Hybrid \\
\hline 11 & Staph. aureus, group-A streptococcus & Bacillus & 72.0 & Cemented \\
\hline
\end{tabular}

* Hybrid, uncemented shell and cemented stem; PFAC, proximal femoral allograft composite; PFR, proximal femoral replacement

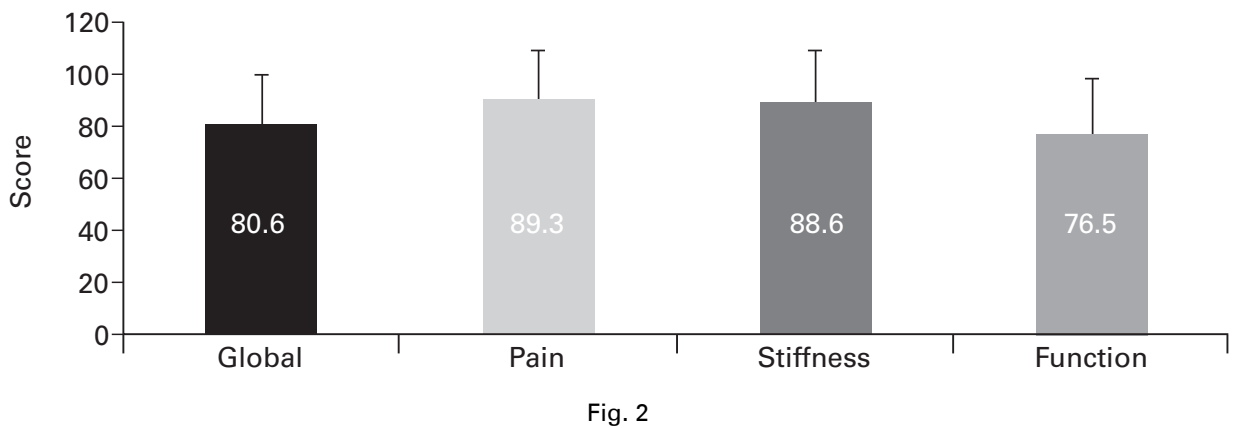

Chart showing the mean WOMAC scores for the Prostalac patients.

\section{Discussion}

In 1970 Bucholz, Elson and Heinert ${ }^{17}$ demonstrated the ability of acrylic cement to elute high concentrations of antibiotics. This concept was initially used in primary joint replacement, but has evolved to include the use of antibiotic-loaded cement in the treatment of periprosthetic infection. ${ }^{18}$ Spacers are formed with antibiotic-loaded cement to deliver high doses of antibiotics at the site of the infection and can achieve local concentrations higher than those obtained with systemic antibiotics alone. Two generic types of antibiotic-impregnated cement depot can be used in two-stage revision THR, namely, non-articulating (typically beads or rods), and articulating spacers. In our experience, articulating spacers promote improved joint function and early mobilisation, maintenance of leg length and retention of tissue planes. The last significantly improves the ease of exposure at the time of re-implantation. The Prostalac system also has a metal endoskeleton giving it inherent strength and thereby reducing the risk of fracture of the spacer before the second stage as well as having a snap-fit socket which reduces the surgical time spent on achieving joint stability at the time of the first stage.

The use of articulated spacers in the first stage of a twostage procedure in the infected THR has been reported to give satisfactory results in the early and mid term. Table III shows that most studies have reported successful eradication of infection in more than $90 \%$ of cases, which is now considered to be the 'gold standard' in North America for the treatment of these difficult cases. ${ }^{19-25}$

Alternatively, a prefabricated spacer such as the Spacer G (Tecres S. pA, Sommacompagna, Verona, Italy $)^{26}$ or the StageOne (Biomet, Warsaw, Indiana) ${ }^{27}$ can be used. Initial results are promising but they are relatively expensive, the dose of antibiotics in the spacer is low and the articulation may cause bone loss in the acetabulum because of the lack of an acetabular component. More recently, Étienne et al, ${ }^{20}$ have shown good results with the use of a modular femoral component or by autoclaving the removed femoral component and mating this with a cemented polyethylene ace- 


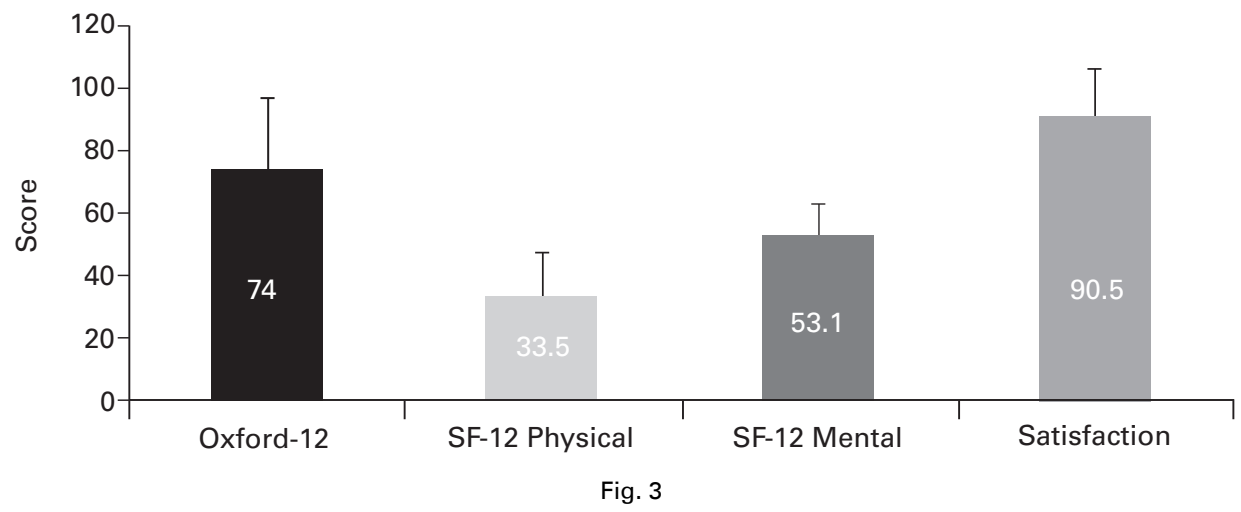

Chart showing the mean Oxford-12, Short-form (SF) -12 and satisfaction scores.

Table III. Review from the literature of the treatment of two-stage infected total hip replacement using an articulated interim antibiotic-laden spacer

\begin{tabular}{|c|c|c|c|c|}
\hline Author/s & Type of spacer & $\begin{array}{l}\text { Number of } \\
\text { patients }\end{array}$ & $\begin{array}{l}\text { Mean follow-up } \\
\text { (mths) }\end{array}$ & $\begin{array}{l}\text { Success rate } \\
(\%)\end{array}$ \\
\hline Younger et al ${ }^{19}$ & Prostalac & 48 & 43 & $45(94.0)$ \\
\hline Etienne et $\mathrm{al}^{20}$ & $\begin{array}{l}\text { Autoclaved femoral component/modular femoral component/polyethylene } \\
\text { acetabular component }\end{array}$ & 32 & 19 & $29(91.0)$ \\
\hline Yamamoto et $\mathrm{al}^{21}$ & Cement moulds with two $2.0 \mathrm{~mm}$ Kirschner wires & 17 & 38 & $17(100.0)$ \\
\hline Durbhakula et $\mathrm{al}^{22}$ & Cement mould with central rod pin & 20 & 38 & $20(100.0)$ \\
\hline Hsieh et $\mathrm{al}^{23}$ & Custom-made cement prosthesis & 42 & 55.2 & $41(97.5)$ \\
\hline Evans $^{24}$ & Low-demand femoral stem/constrained polyethylene liner & 23 & 24 & $22(95.7)$ \\
\hline Hofmann et al ${ }^{25}$ & $\begin{array}{l}\text { Autoclaved femoral component/modular femoral component/polyethylene } \\
\text { acetabular component }\end{array}$ & 27 & 76 & $26(94.0)$ \\
\hline
\end{tabular}

tabular liner. They rely on coating the femoral component with a layer of cement, but in our experience this can be an onerous and a time-consuming task. Others have used spacers with Rush pins or Kirschner-wires as the metal endoskeleton with success, but again the rigidity cannot be relied upon. . $^{2-23}$

In our study the rate of eradication of the initial infection was $89 \%$ with a two-stage approach using the articulated Prostalac spacer system. With further surgery (usually an added exchange) the final success rate rose to $96 \%$. In five of the 11 patients with re-infection another organism was cultured. The potential implication is that the causative organism had been eradicated and the success rate was greater than that observed. Alternatively, the re-infecting organisms may have been present from the beginning, but not initially isolated. Five of the re-infections recurred within a year of the second stage and were successfully treated by further surgery. Our results show that this method can lead to the sustained long-term eradication of infection with good quality-of-life outcomes and, to the best of our knowledge, this is the largest series reported on the use of articulated spacers in the two-stage treatment of infection of the hip with a minimum follow-up of ten years.

There has been much controversy regarding the directexchange technique or a delayed reconstruction for infection. Single-stage exchange revision using antibiotic-loaded cement was first popularised in Europe and has been recommended in immune-competent patients with an acute infection, sensitive to first-line antibiotics. ${ }^{5,6}$ The main disadvantage of this technique is the need to use antibioticloaded cemented fixation at the single stage, which may compromise the longevity of the implant since cemented femoral and acetabular components have not been shown to be as durable as cementless implants in revision THR. ${ }^{28-30}$ With the increasing emergence of antibiotic-resistant bacteria and an increased prevalence of revision arthroplasties with associated bone loss requiring bone grafting, the feasibility of direct exchange currently is limited. Several reports have shown success with direct exchange, but these have been selective. ${ }^{5,31}$ The only study which was not selective was that by Raut et $\mathrm{al}^{6}{ }^{6}$ but these results have not been reproduced to date. A number of studies comparing singlestage versus two-stage exchange all favoured the two-stage procedure. ${ }^{29,32}$ Although analyses based on combined series have major biases, delayed reconstruction appears to provide the highest rate of success.

Another controversial aspect in the management of these patients is the use of antibiotics, their delivery and the duration of treatment after the first-stage procedure. Lieberman et $\mathrm{al}^{7}{ }^{7}$ and Salvati et $\mathrm{al}^{8}{ }^{8}$ suggested that there should be a six-week course of parenteral antibiotics after the first stage. However, recently it was shown that the use 
of a two-stage exchange procedure with antibiotic-loaded cement and without parenteral antibiotics, gave a rate of eradication of $87.7 \% .{ }^{34}$ This highlights the concept that the delivery of local antibiotics is paramount and that the associated costs of long-term parenteral antibiotics can be minimised by reducing the duration of treatment or potentially even eliminating them from the protocol, as long as an antibiotic-loaded spacer is used. More recently, Winkler et al ${ }^{35}$ have shown that using antibiotic-impregnated cancellous allograft bone in one-stage uncemented hip revisions for infection can provide high rates of eradication in the short term. These concepts need further analysis and may yield significant implications for the management of these cases.

When assessing patient-orientated outcomes, Scharfenberger et $\mathrm{al}^{36}$ investigated the treatment of infected THR, with the Prostalac system and reviewed the function and health-related quality-of-life of 23 patients at follow-up at two years using the WOMAC index score and the Harris hip score. They concluded that the Prostalac implant allowed patients to have a reasonable function and qualityof-life during the interim treatment for deep infection. Our study did not determine quality-of-life scores in the interval stage with the Prostalac hip spacer, but at the long-term follow-up after the second stage these scores were good and satisfaction was extremely high with a mean score of 90.5. When considering success, the outcome is multifactorial and the durability of the reconstruction is important. Our study has shown that the re-operation rate for reasons not related to infection was modest at $9 \%$ and was comparable with that of other studies. ${ }^{37,38}$

The strength of our study was that we were able to gather reliable information on 92 patients $(93 \%)$ of the series, since all complications were referred back to our tertiary referral centre. This combined with extensive tracking and follow-up allowed us to make a valid interpretation of the outcome. The limitations were that only 34 patients returned quality-of-life outcome scores and this may not have truly represented the group as a whole. However, the distribution of the Charnley class was similar to that of the original series and therefore potentially showed a similar trend. We were not able to perform a comprehensive radiological review since most patients had good function with no clinical evidence of infection and were often located far from our tertiary centre. All current radiographs at our institution did not show any evidence of failure of the implant; including the subset of patients who sent in updated radiographs (10 of 48). Inflammatory markers were only requested if there was a clinical suspicion of infection but of the 48 patients still alive most had good function and these tests were not undertaken.

In conclusion, our findings showed that two-stage revision with an intermediate Prostalac hip spacer was a safe and effective means of treating an infected THR with a long-term success rate of $89 \%$. An added exchange in a small number of relcalcitrant cases allowed the success rate to rise to $96 \%$.
There were good levels of pain control, function and satisfaction with the use of this method.

No benefits in any form have been received or will be received from a commercial party related directly or indirectly to the subject of this article.

\section{References}

1. Masterson EL, Masri BA, Duncan CP. Treatment of infection at the site of total hip replacement. Instr Course Lect 1998;47:297-306.

2. Pagnano MW, Trousdale RT, Hanssen AD. Outcome after reinfection following reimplantation hip arthroplasty. Clin Orthop 1997;338:192-204.

3. McDonald DJ, Fitzgerald RH Jr, Ilstrup DM. Two-stage reconstruction of a total hip arthroplasty because of infection. J Bone Joint Surg [Am] 1989;71-A:828-34.

4. Kilgus DJ, Howe DJ, Strang A. Results of periprosthetic hip and knee infections caused by resistant bacteria. Clin Orthop 2002;404:116-24

5. Callaghan JJ, Katz RP, Johnston RC. One-stage revision surgery of the infected hip: a minimum 10-year followup study. Clin Orthop 1999;369:139-43.

6. Raut VV, Orth MS, Orth MC, Siney PD, Wroblewski BM. One stage revision arthroplasty of the hip for deep gram negative infection. Int Orthop 1996;20:12-14.

7. Lieberman JR, Callaway GH, Salvati EA, et al. Treatment of the infected total hip arthroplasty with a two-stage reimplantation protocol. Clin Orthop 1994;301:205-12.

8. Salvati EA, Chekofsky KM, Brause PD, Wilson D Jr. Reimplantation in infection: a 12-year experience. Clin Orthop 1982;170:62-75.

9. Whittaker JP, Warren RE, Jones RS, Gregson PA. Is prolonged systemic antibiotic treatment essential in two stage revision hip replacement for chronic Grampositive infection? J Bone Joint Surg [Br] 2009;91-B:44-51.

10. Bellamy N, Buchanan W, Goldsmith CH, Campbell J, Stitt LW. Validation study of WOMAC: a health status instrument for measuring clinically important patient relevant outcomes to antirheumatic drug therapy in patients with osteoarthritis of the hip and the knee. J Rheumato/ 1988;15:1833-40.

11. Dawson J, Fitzpatrick R, Carr A, Murray D. Questionnaire on the perceptions of patients about total hip replacement. J Bone Joint Surg [Br] 1996;78-B:185-90.

12. Ware JE, Sherbourne CD. The MOS 36-item Short-Form Health Survey SF-36). Med Care 1992;30:473-83.

13. Amstutz HC, Thomas BJ, Jinnah R, et al. Treatment of primary osteoarthritis of the hip: a comparison of total joint and surface replacement arthroplasty. J Bone Joint Surg [Am] 1984;66-A:228-41.

14. Solomon DH, Bates DW, Horsky J, et al. Development and validation of a patient satisfaction scale for musculoskeletal care. Arthritis Care Res 1999;12:96100

15. Charnley J. The long-term results of low-friction arthroplasty of the hip performed as a primary intervention. J Bone Joint Surg [Br] 1972;54-B:61-76.

16. McFarland B, Osborne G. Approach to the hip: a suggested improvement on Kocher's method. J Bone Joint Surg [Br] 1954;36-B:364-7.

17. Bucholz HW, Elson RA, Heinert K. Antibiotic loaded acrylic cement: current concepts. Clin Orthop 1984;109:96-108.

18. Webb JC, Spencer RF. The role of polymethylmethacrylate bone cement in modern orthopaedic surgery. J Bone Joint Surg [Br] 2007;89-B:851-7.

19. Younger AS, Duncan CP, Masri BA, et al. The outcome of two-stage arthroplasty using a custom-made interval spacer to treat the infected hip. J Arthroplasty 1997; 12:615-23

20. Etienne G, Waldman B, Rajadhyaksha AD, Ragland PS, Mont MA. Use of a functional temporary prosthesis in a two-stage approach to infection at the site of a total hip arthroplasty. J Bone Joint Surg [Am] 2003;85(Suppl 4):94-6.

21. Yamamoto K, Miyagawa N, Masaoka T, et al. Clinical effectiveness of antibiotic-impregnated cement spacers for the treatment of infected implants of the hip joint. J Orthop Scie 2003;8:823-8.

22. Durbhakula SM, Czajka J, Fuchs MD, UhI RL. Spacer endoprosthesis for the treatment of infected total hip arthroplasty. J Arthroplasty 2004;19:760-7.

23. Hsieh PH, Chen LH, Chen CH, et al. Two-stage revision hip arthroplasty for infection with a custom-made, antibiotic-loaded, cement prosthesis as an interim spacer. J Trauma 2004:56:1247-52.

24. Evans RP. Successful treatment of total hip and knee infection with articulating antibiotic components: a modified treatment method. Clin Orthop 2004;427:37-46.

25. Hofmann AA, Goldberg TD, Tanner AM, Cook TM. Ten-year experience using an articulating antibiotic cement hip spacer for the treatment of chronically infected total hip. J Arthroplasty 2005;20:874-9

26. Magnan B, Regis D, Biscaglia R, Bartolozzi P. Performed acrylic bone cement spacer loaded with antibiotics. Acta Orthop Scand 2001;72:591-4 
27. Griffin SJ, Steel NA. The use of the biomet femoral spacer in stages revision for infection of the total hip arthroplasty. J Bone Joint Surg [Br]2004;86-B(Suppl 1):72.

28. Moreland JR, Moreno MA. Cementless femoral revision arthroplasty of the hip: minimum 5 years followup. Clin Orthop 2001;393:194-201.

29. Weeden SH, Paprosky WG. Porous-ingrowth revision acetabular implants secured with peripheral screws: a minimum twelve-year follow up. J Bone Joint Surg [Am] 2006;88-A:1266-71.

30. McLaughlin JR, Harris WH. Revision of the femoral component of a total hip arthroplasty with the calcar-replacement femoral component: results after a mean of 10.8 years postoperatively. J Bone Joint Surg [Am] 1996;78-A:331-9.

31. Nagai H, Wroblewski B, Gambbir AK, Kay PR, Siney PD. One stage revision total hip replacement for deep infection: 5- to 27-year follow-up study. Procs 70th Annual Meeting American Academy of Orthopaedic Surgeons, 2003.

32. Elson RA. Exchange arthroplasty for infection: perspectives from the United Kingdom. Orthop Clin North Am 1993;24:761-7.
33. Garvin KL, Fitzgerald RH Jr, Salvati EA, et al. Reconstruction of the infected total hip and knee arthroplasty with gentamicin-impregnated Palacos bone cement. Instr Course Lect 1993;42:293-302.

34. Stockley I, Mockford BJ, Hoad-Reddick A, Normal P. The use of two-stage exchange arthroplasty with depot antibiotics in the absence of long-term antibiotic therapy in infected total hip replacement. J Bone Joint Surg [Br] 2008;90-B:145-8.

35. Winkler H, Stoiber A, Kaudela K, Winter F, Menschik F. One stage uncemented revision of infected total hip replacement using cancellous allograft bone impregnated with antibiotics. J Bone Joint Surg [Br] 2008;90-B:1580-4.

36. Scharfenberger A, Clark M, Lavoie G, et al. Treatment of an infected total hip replacement with the PROSTALAC system. Part 2: health-related quality of life and function with the PROSTALAC implant in situ. Can J Surg 2007;50:29-33.

37. Sanchez-Sotelo J, Berry DJ, Hanssen AD, Cabanela ME. Midterm to long-term followup of staged reimplantation for infected hip arthroplasty. Clin Orthop 2009;467:219-24.

38. English H, Timperley AJ, Dunlop D, Gie G. Impaction grafting of the femur in twostage revision for infected total hip replacement. J Bone Joint Surg [Br] 2002;84-B:700-5. 